\title{
Gorlin-Goltz syndrome with familial manifestation
}

\author{
Jindřich Pazdera ${ }^{\mathrm{a}}$, Alena Santavab ${ }^{\mathrm{b}}$ Zdenek Kolarc
}

\begin{abstract}
Aims. The detection of odontogenic keratocysts (OKC) in the oral cavity is one of the main criteria for the clinical manifestation of Gorlin-Goltz syndrome (Nevoid Basal Cell Carcinoma Syndrome - NBCCS). From a clinical point of view, we distinguish between "syndromic" and "sporadic" OKC. Syndromic cysts, often multifocal, may be an accidental finding on X-ray examination. They can manifest gradually depending on the development of permanent dentition. Sporadic cysts are rather solitary lesions with clinical manifestation in adulthood.

Methods. Mutations in the PTCH1 gene are thought to be the cause of the clinical manifestation of NBCCS. These abnormalities can be transmitted from one generation to another and lead to a familial occurrence of the disease. In $35-50 \%$ of cases, these are a newly arising mutations. It is necessary to take into account the typical manifestations which in the next generation begin at a younger age and the disease usually has a more serious course.

Results. We found a familial manifestation of NBCCS in two pairs of patients (mother and daughter and two siblings). Odontogenic keratocysts and cutaneous basal cell carcinomas were diagnosed and genetic testing revealed mutations in the PTCH 1 gene in all four individuals.

Conclusions. With regard to the possibility of familial occurrence of NBCCS, it is necessary to pay increased attention to family history and, if necessary, to ensure clinical and genetic examination of parents and other family members. Patients of childbearing potential with evidence of NBCCS should be informed of the increased likelihood of the disease in the offspring.
\end{abstract}

Key words: odontogenic keratocysts, Gorlin-Goltz syndrome (NBCCS), familial manifestations, genetic testing

Received: September 16, 2020; Revised: November 25, 2020; Accepted: December 8, 2020; Available online: February 4, 2021 https://doi.org/10.5507/bp.2020.063

(c) 2022 The Authors; https://creativecommons.org/licenses/by/4.0/

${ }^{a}$ Department of Oral and Maxillofacial Surgery, Faculty of Medicine and Dentistry, Palacky University Olomouc, Czech Republic ${ }^{b}$ Department of Medical Genetics, Faculty of Medicine and Dentistry, Palacky University Olomouc, Czech Republic

'Department of Pathology, Faculty of Medicine and Dentistry, Palacky University Olomouc, Czech Republic Corresponding author: Jindrich Pazdera, e-mail: Jindrich.Pazdera@fnol.cz

\section{INTRODUCTION}

Nevoid Basal Cell Carcinoma Syndrome (NBCCS, Gorlin-Goltz syndrome) is an autosomal dominant inherited disease with complete penetration and variable expression. In its clinical manifestation, new dominant mutations of the PTCH1 gene are involved in $35-50 \%$ of cases $^{1}$. The gene, the product of which is a transmembrane protein of the Patched family, is located in the long arm region of chromosome 9 (9q22.1 - q31). As a tumour suppressor, it inhibits the expression of genes encoding signal proteins belonging to the TGF-beta and Wnt families. Inactivation of both gene alleles appears to lead to tumor formation, while developmental and skeletal abnormalities, which are also caused by the gene defect, appear to be due to the presence of a single functional allele. The prevalence of NBCCS is approximately 1 case per 60,000 people.

Our diagnosis of NBCCS is based on the occurrence of at least two main or one major and two minor criteria (Tab. 1). From a dental point of view, the disease is interesting mainly because it is accompanied by odontogenic keratocysts of the jaw bones and basal cell carcinomas on the skin of the face, neck and chest. The shape of the head and the configuration of the face are also typical (macrocephaly with prominent frontal region and hypertelorism) $\left(\right.$ ref. $\left.^{2}\right)$.

\section{CASE REPORT 1}

Mother (born 1947) has been treated in the Department of Oral, Maxillofacial and Facial Surgery since 2005. The immediate reason for starting the treatment was the clinical manifestation of multiple basal cell carcinomas in the face and hair of the head and trunk. The shape of the patient's head and face configuration is similar to that of her daughter, typical of Gorlin's syndrome: macrocephaly, hypertelorism, a more robust figure. In her case history, recorded is surgery of a jaw cyst in childhood (histological findings could not be traced). At the age of 33, the first facial basal cell carcinomas were removed at various other sites. The patient's mother had similar problems (jaw cyst, basal cell carcinoma).

During the treatment, in addition to multiple basal cell carcinomas in the face, hair of the head and trunk, a large cystic lesion of the right angle area and branch of the mandible was also diagnosed (Fig. 1). With regard to the size of the pathological cavity, we chose fenestration followed by long-term drainage into the oral cavity. We 


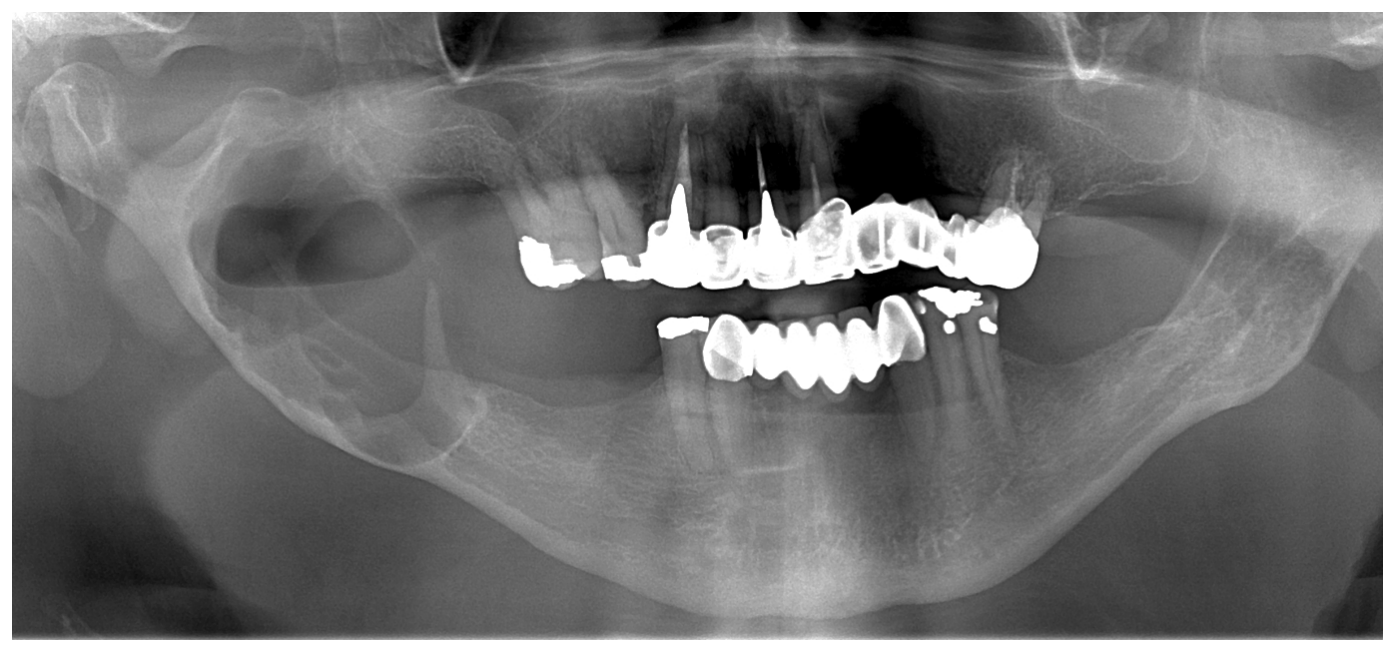

Fig. 1. Large odontogenic keratocyst of the right mandibular angle.

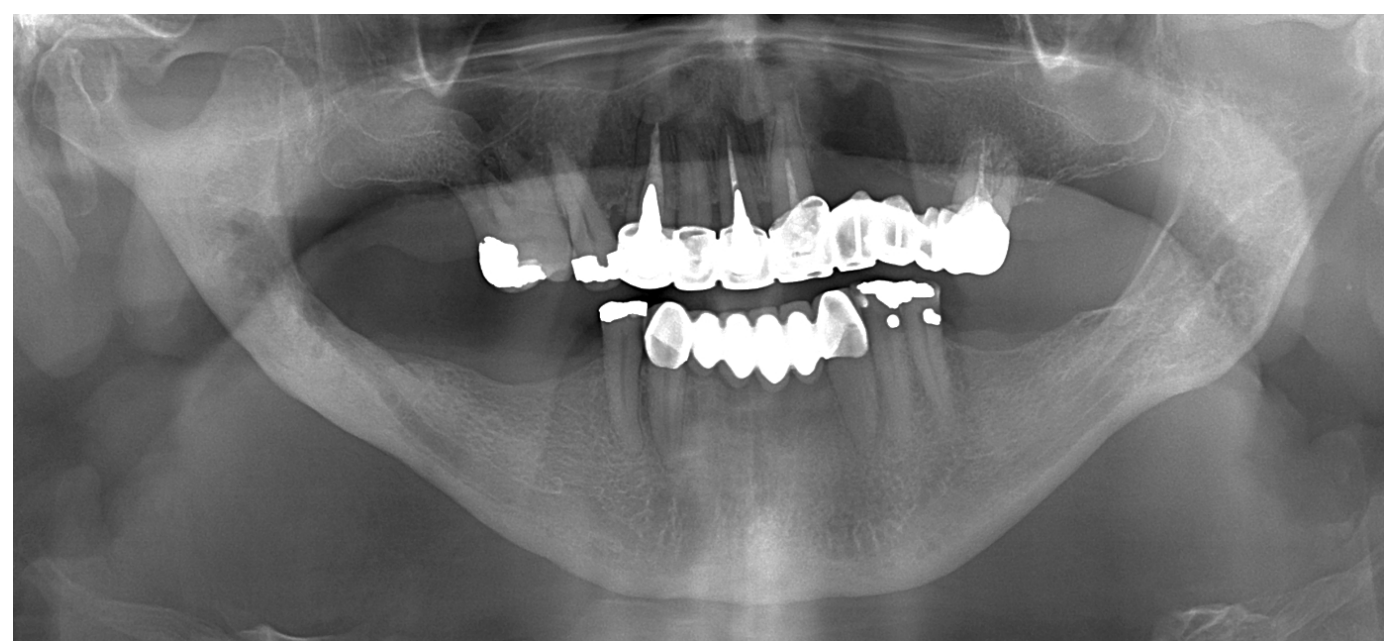

Fig. 2. X-ray finding 18 months after decompression and drainage of cystic cavity.

performed a surgical revision 18 months later - after the defect was reduced (Fig. 2). The histopathology revealed fragments of muscle-fat tissue permeated by fibrosis with chronic inflammatory cellulitis, some fragments had the appearance of non-specific granulation tissue with mixed inflammation. In one of the blocks, the tissue had, in places a cystic configuration, lined with squamous epithelium with superficial parakeratosis and marked palisading of basal cells. The inner surface of the cyst had an undulating course. This finding was consistent with the diagnosis of odontogenic keratocyst.

Daughter (born 1967) has been treated in the Department of Oral, Maxillofacial and Facial Surgery, Olomouc University Hospital, since 1989 for recurrent odontogenic keratocysts located in both angles of the mandible. Multiple facial basal cell carcinomas also appeared several years later. Macrocephaly and typical face with hypertelorism were noticeable. There was scoliosis of the spine. CT of the brain showed calcification of the falx cerebri. Ovarial tumours were not detected; condition after hysterectomy for uterine fibroids.

Recurrent, histologically verified odontogenic keratocysts in both angles of the mandible were repeatedly extirpated during treatment. In the case of postoperative recurrences, Carnoy's solution was applied to the bone cavity at the end of the surgery. In 2016, a newly discovered odontogenic keratocyst in the left maxilla was extirpated. Gradually, small basal cell carcinomas were excised.

DNA analysis of both patients revealed c.2179delT mutation (p.Cys727ValfxX19) in the PTCH gene in a heterozygous form. This is a clearly confirmed familial occurrence of a Gorlin-Goltz syndrome (autosomal dominant inheritance). The case is characterized by anticipation - when the manifestations in the daughter began at a younger age and the disease had a more serious course.

\section{CASE REPORT 2}

Older brother (born 1984) was treated in our department from the age of 13 to 30 for multiple skin basal cell carcinomas and recurrent keratocysts of the upper and lower jaw with multifocal occurrence. His mother and grandmother also had similar problems, so does his younger brother. 
Table 1. Gorlin-Goltz syndrome - diagnostic criteria.

\begin{tabular}{ll}
\hline Major criteria & Minor criteria \\
\hline $\begin{array}{l}\text { More than } 2 \text { basal cell carcinomas under } \\
\text { the age of } 20 \text { years }\end{array}$ & Macrocephaly \\
Odontogenic keratocysts of the jaw bones & Frontal bossing, hypertelorism \\
Three or more palmar keratotic pittings & Cleft lip and palate \\
Calcification of falx cerebri & Ovarial tumors \\
Skeletal deformities & Medulloblastoma \\
Confirmed NBCCS in parents & \\
\hline
\end{tabular}

At the entrance clinical examination, hypertelorism, macrocephaly and prominent eyelid arches, chest skeletal deformities, and isolated palmar depressions were noticeable.

The reason for starting the treatment was a large cyst in the lower jaw area attached to the unerupted right lower canine. During the development of dentition, keratocysts were gradually manifested in the area of both angles of the mandible (on the left again associated with the unerupted third molar) and in the area of the right maxillary sinus. Over several, we also surgically removed several small basal cell carcinomas. The diagnosis of odontogenic keratocysts was confirmed by histological examination.

Younger brother (born 1987) was treated at the Department of Oral, Maxillofacial and Facial Surgery from the age of 21 to 23 for odontogenic keratocysts with multifocal manifestation and associated with unerupted third molars in both angles and branches of the mandible and both maxillary cavities and multiple minor facial skin basal cell carcinomas (Fig. 3, 4). The shape of the head and the configuration of his face were similar to those of his older brother. During treatment, we gradually extirpated cysts at both angles of the mandible and in both maxillary cavities and extracted uerupted teeth.

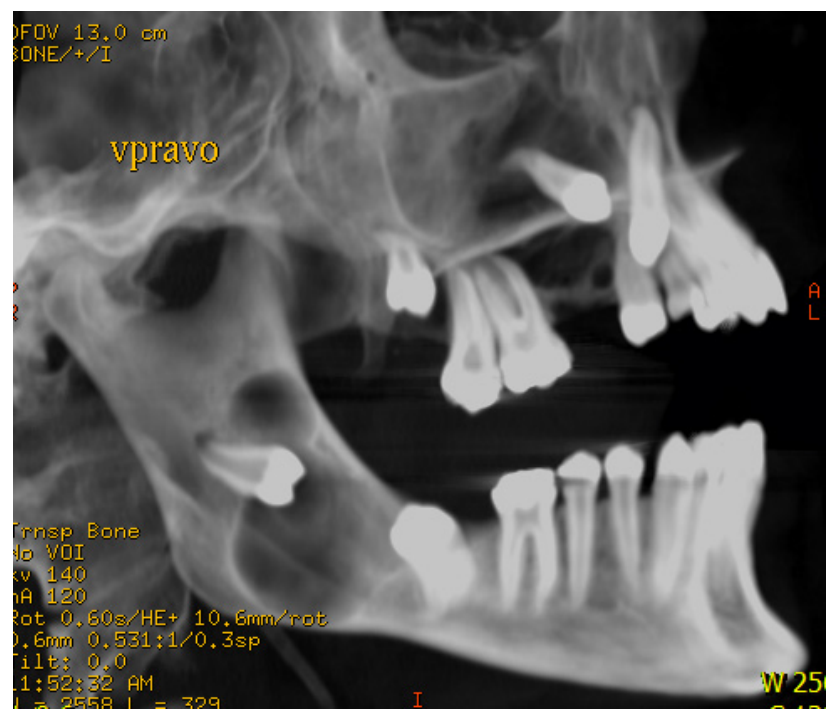

Fig. 3. Multiple syndromic keratocysts associated with retained teeth.
DNA examination of both siblings revealed a c.655-1G A mutation in the PTCH1 gene, confirming the diagnosis of Gorlin's syndrome. Despite our recommendations, both brothers stopped attending the regular outpatient check-ups and did not respond to written invitations.

\section{RESULTS}

We diagnosed the Gorlin-Goltz syndrome in five other patients treated at our department. In these cases too, there was most likely a new dominant mutation. What prevailed here were clinical manifestations of odontogenic keratocysts and skin basal cell carcinomas. The number of cystic lesions in our patient group varied from one to four. Among the secondary criteria, macrocephaly with hypertelorism, forehead arching, and typical facies predominated. Of the skeletal abnormalities, we observed chest skeletal deformity in three patients and falx cerebri calcification in the other two.

When choosing surgical procedures, we opted for extirpation of cystic lesions. In case of postoperative recur-

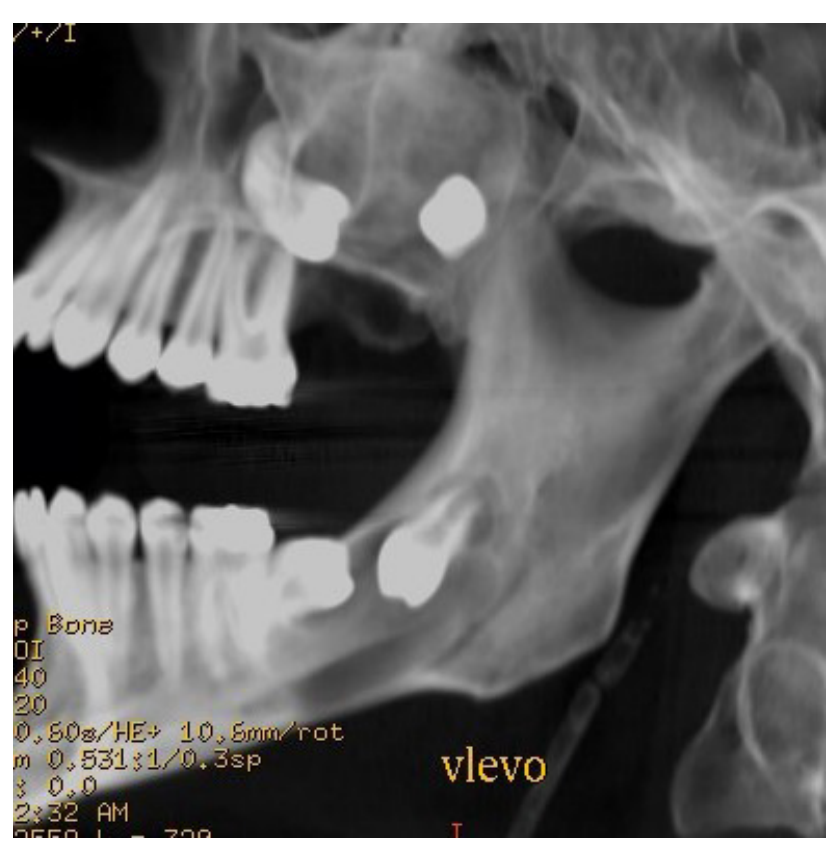

Fig. 4. Multiple syndromic keratocysts associated with retained teeth. 
rences after extirpation and histological verification, we treated the residual bone cavity with Carnoy's solution applied on a gauze tampon for $2 \mathrm{~min}$.

A long-termed follow up of all our patients with proven NBCCS is necessary. We perform the detection of skin basal cell carcinomas twice a year, and we make radiographs of the jaw bones once a year.

\section{DISCUSSION}

NBCCS is an autosomal dominant disease characterized by developmental disorders and the development of tumours (Table 1). The cause of the clinical manifestation of NBCCS is a mutation in the PTCH1 gene for the Patched receptor ${ }^{1,3-5}$. Basal cell carcinomas, astrocytomas, ovarian carcinomas or medulloblastomas may occur in $3-4 \%$ of patients with NBCCS. In these cases, deletion of 9q22.1-31 is accompanied by loss of gene heterozygosity on the homologous chromosome. These genetic defects can be passed on from one generation to the next and are manifested by a familial occurrence of the disease. In $35-$ $50 \%$ of cases, these are new mutations. From a medical point of view, it is necessary to anticipate manifestations of the syndrome with an earlier onset and a more serious course in the next generation. Patients who expect to have children should be aware of the $50 \%$ risk of this disease in their offspring. Therefore, genetic testing is recommended to all patients with clinical manifestations of at least two main NBCCS criteria. In these patients, a history of the disease in grandparents, parents, children or siblings should be investigated. Living close relatives should be examined for evidence of clinical manifestations of NBCCS.

The occurrence of odontogenic keratocysts is one of the main diagnostic criteria for NBCCS. These cysts are thought to derive from the remnants of dental lamina. Their biological behavior is similar to benign neoplasmas. The literature shows that these lesions are often diagnosed in patients in the second decade of life. The mandible is affected more often than the maxilla ${ }^{6,7}$. The predilection localization in the mandible is the area of the mandibular angle, in the upper jaw there is frequent propagation to the maxillary cavities, associated with long, asymptomatic growth. Cysts, which may or may not always be attached to an unerupted tooth, reach considerable size but can be clinically manifested relatively late. This is due to the nature of their growth, which is oriented mainly in the posteroanterior dimension. Therefore, the typical bone deformation, characteristic eg for radicular cysts, is missing.

From the histopathological point of view, OKCs are characterized by a very thin lining formed by a layered squamous epithelium with signs of parakeratosis, the basal layer of which has a typical palisade-like arrangement. The surface of the lining towards the lumen is uneven, undulating. Satellite microcysts are usually present in the connective tissue of the capsule.

In connection with the clinical manifestation of NBCCS, some authors distinguish between "sporadic" and "syndromic" keratocysts ${ }^{8-11}$. "Syndromic" keratocysts with NBCCS binding are characterized by an increased number of microscopic satellite cysts (greater tendency to recurrence) and the presence of solid islets of epithelial proliferation in the walls with increased mitotic lining activity. "Syndromic" keratocysts are usually manifested in younger individuals. We have a similar experience from our own practice: we diagnosed most "syndromic" cysts in individuals in the second age decade. It was mainly a multifocal occurrence and a gradual clinical manifestation over several years, linked to the development of permanent dentition. Syndromic keratocysts also have a different immunophenotype compared with sporadic OKCs. These are characterized by higher expression of $\mathrm{Bcl}-2$, p27Kip 1 are nd c-erbB-2 and lower proliferative activity in epithelial basal cells along with a low inflammatory response. OKC differs from other odontogenic cysts by a different regulation of apoptosis and cell proliferation ${ }^{12}$.

Syndromic OKC are characterized by a higher tendency to postoperative recurrences (30-60\%). The causes are various: incomplete removal of the thin, fragile sac, satellite microscopic cysts in the fibrous tissue of the sheath, or remnants of the dental lamina, persisting in the wall of the residual bone cavity.

At present, it can be stated that odontogenic keratocysts are not a diagnostic problem, but rather a therapeutic problem. The orthopantomogram, as a common part of the patient's initial dental examination which allows us, often accidentally, to diagnose clinically asymptomatic cystic lesions. The problem remains the choice of the optimal therapeutic procedure that will minimize the high probability of postoperative recurrences. Postoperative recurrences are associated with a small risk of malignant reversal of the cystic sac lining into spinocellular carcinoma ${ }^{13-16}$. We ourselves have observed one similar case in the past in a 50-year-old man with a malignant reversal of recurrent $\mathrm{OKC}$, unfortunately with a lethal end. Transformation of OKC into a cystic form of ameloblastoma is also possible.

Another factor is the choice of the optimal therapeutic procedure of $\mathrm{OKC}$. Because simple cyst enucleation is associated with a high probability of postoperative recurrence, it is recommended to modify the surgical procedures in various ways. Probably the oldest option is perioperative application of corrosive Carnoy's solution to the residual bone cavity ${ }^{17}$. In our experience, this method is not completely reliable, moreover, the chloroform contained in the solution is considered to be a carcinogen. Another option is to mill the walls of the residual bone cavity which can be used for smaller or medium-sized cysts. Radical resection of the affected part of the jaw skeleton with subsequent reconstruction with a bone graft is controversial in children with incomplete growth and development of the jaws and difficult to use in multifocal syndrome lesions. Fenestration of the cystic cavity with long-term drainage seems to be promising for the treatment of large cysts, and can lead to a remarkable reduction in the volume of the cystic cavity in a number of months and perhaps even a change in biological properties towards lower aggressiveness ${ }^{18-22}$. Classical marsupialization can also be applied. The percentage of 
postoperative recurrences with this method of treatment is significntly lower, as demonstrated by Chinese authors ${ }^{23}$ in a large group of patients. According to our (so far limited) experience, perioperative augmentation of residual bone cavities with ground autologous cancellous bone or allogenic material with osseoinductive effect can also bring promising results.

In connection with the high tendency of "syndromic" OKCs to postoperative recurrences, the question of early detection of postoperative relapses remains open. Because it is recommended that radiation exposure be reduced as much as possible in patients with NBCCS, radiographs of the jawbones should not be performed frequently. In our opinion, an X-ray once every 12 months is enough.

Multiple basal cell carcinomas appear only rarely in childhood; their occurrence is more frequent in puberty and adulthood. At the beginning, they have the character of small papules which are concentrated in the face, hairy part of the head, neck and chest. In the postpubertal period, their behaviour may be more aggressive. Other cutaneous manifestations include numerous milia, epidermoid cysts, comedomes, plantar and palmar keratotic pitting.

\section{CONCLUSION}

NBCCS is a systemic disease that can be indirectly noticed by a dentist by the identification of odontogenic keratocysts, the manifestations of which are among the main criteria for this syndrome. From a therapeutic point of view, given the high probability of postoperative recurrences, it is necessary to modify the surgical approach, and to do a complete clinical examination of the patient focused on possible demonstration of other criteria confirming the clinical manifestations of NBCCS. Postoperative follow-up cannot be underestimated. Given the familial manifestation of the disease, it is necessary to pay attention to family history, genetic and clinical examination of family members.

Author contributions: JP: literature search; JP AS, ZK: manuscript writing, data analysis; JP, ZK: final approval. Conflict of interest statement: The authors state that there is no conflict of interest regarding the publication of this article.

\section{REFERENCES}

1. Özcan G, Balta B, Sekerci AE, Etoz OA, Martinuzzi C, Kara O, Pastorino L, Kocoglu F, Ulker O, Erdogan M. A novel PTCH1 gene mutation in pediatric patient associated multiple keratocystic odontogenic tumors of the jaws and Gorlin-Goltz syndrome. Indian J Pathol Microbiol 2016;59:335-8.
2. Gorlin RJ, Goltz R. Multiple nevoid basal cell epitheliomata, jaw cysts, bifid rib-A syndrome. N Eng J Med 1960;262:9008-911.

3. Lazaridou MN, Katopodi T, Dimitrakopoulos I. Gorlin-Goltz syndrome: a 25 years follow-up of a familiar case. Oral Maxillofac Surg 2015; 19:79-84.

4. Fujii $M$, Noguchi $K$, Urade $M$, Muraki Y, Kishimoto $H$, HashimotoTamaoki T, Nakano Y. Novel PTCH1 mutations in Japanese Nevoid basal cell carcinoma syndrome patients. J Hum Genet 2011;56(4):277-83.

5. Lam CW, Leung CY, Lee KC, Xiei J, Lo FM, Au ST, Tong SF, Pon MK Chan LY, Luk NM. Novel mutations in the PATCHED gene in basal cell nevus syndrome. Mol Genet Metab 2002;76(1):57-61.

6. Güven O, Keskln A, Akal ÜK. The incidence of cysts and tumors around impacted third molars. Int J Oral Maxillofac Surg 2000;29(2):131-35.

7. Stoelinga PJW, Bronkhorst FB. The incidence, multiple presentation and recurrence of agressive cysts of the jaws. J Craniomaxillofac Surg 1988;16:184-95.

8. Dominguez FR, Keszler A. Comparative study of keratocysts associated and nonassociated with nevoid basal cell carcinoma syndrome. J Oral Pathol 1988;17:39-42.

9. Shear M. The aggressive nature of the odontogenic keratocysts: is it a benign cystic neoplasm? Part 1: Clinical and early experimental evidence of aggressive behaviour. Oral Oncol 2002;38:219-26.

10. Shear $M$. The aggressive nature of the odontogenic keratocyst: is it a benign cystic neoplasm? Part 2: Proliferation and genetic studies. Oral Oncol 2002;38:323-31.

11. Noy D, Rachmiel A, Zar K, Emodi O, Nagler RM. Sporadic versus syndromic keratocysts- Can we predict treatment outcome? A review of 102 cysts. Oral Diseases 2017;23(8):1058-66.

12. Kolář Z, Geireová M, Bouchal J, Pazdera J, Zbořil V, Tvrdy P. Immunohistochemical analysis of the biological potential of odontogenic keratocysts J Oral Pathol Med 2006;35(2):75-80.

13. Keszler A, Piloni MJ. Malignant transformation in odontogenic keratocyst. Case report. Med Oral 2002;7(5):331-35.

14. Yoshida $\mathrm{H}$, Onizawa $\mathrm{K}$, Yusa $\mathrm{H}$. Squamous cell carcinoma arising in association with an orthokeratinized odontogenic keratocyst Report of a case. J Oral Maxillofac Surg 1996;54:647-51.

15. Makowski GJ, McGuff S, Van Sickels JE. Squamous cell carcinoma in a maxillary odontogenic keratocysts. J Oral Maxillofac Surg 2001;59:76-80.

16. Murillo-Cortes J, Pérez AE, López CS, Gorbea RM, Rodriguez-Cortel JM. Primary intraosseous carcinoma arising in mandibular cyst. Med Oral 2002;7(5):370-74.

17. Voorsmit RACA. The art of treating keratocysts: fixation before enucleation. Autumn Meeting of the British Assoc of Oral and Maxillofacial Surgeons London 1990

18. August M, Faquin WC, Troulis MJ, Karban LB. Dedifferentiation of odontogenic keratocyst epithelium after cyst decompression. J Oral Maxillofac Surg 2003;61(6):678-83.

19. Wushou A, Zhao YJ, Shao ZM. Marsupialization is the optimal treatment approach for keratocystic odontogenic tumour. J Craniomaxillofac Surg 2014;42(7):1540-44.

20. Ninomiya T, Kubota Y, Koji T, Shirasuna K. Marsupialization inhibits interleukin-1 alpha expression and epithelial cell proliferation in odontogenic keratocysts. J Oral Pathol Med 2002;31(9):526-33.

21. Blanas N, Freund B, Schwartz M, Furst IM. Systemic review on the treatment and prognosis of the odontogenic keratocysts. Oral Surg Oral Med Oral Pathol Oral Radiol Endod 2000;90:553-58.

22. Tolstunov L, Treasure T. Surgical treatment algorhytm for odontogenic keratocysts: Combined treatment of odontogenic keratocyst and mandibular defect with marsupialization, enucleation, iliac crest bone graft, and dental implants. J Oral Maxillofac Surg 2008;66:102536.

23. Zhao YF, Wei JX, Wang SP. Treatment of odontogenic keratocysts: a follow up of 255 Chinese patients. Oral Surg Oral Med Oral Pathol Oral Radiol Endod 2002;92(2):151-56. 